\title{
Melanoma Recurrence After Mohs Micrographic Surgery with MART-1: A Systematic Review and Meta-Analysis
}

\author{
Tyler Long, DO ${ }^{1 *}$, Austin Dunn, $\mathrm{DO}^{2}$, Mary Arndt, $\mathrm{MS}^{3}$, Eric Parlette, $\mathrm{MD}^{1}$ \\ ${ }^{1}$ HCA Healthcare LewisGale Hospital-Montgomery, Blacksburg, VA \\ 2 Center for Clinical and Cosmetic Research, Aventura, FL \\ ${ }^{3}$ Lake Erie College of Osteopathic Medicine, Bradenton, FL
}

\section{ABSTRACT}

Background: Surveys suggest wide-spread use of MART-1 in MMS for CM among Mohs surgeons, but no previous systematic review has investigated the efficacy of this specific procedure.

Methods: A systematic search and meta-analysis of retrospective studies on MMS with MART-1 was performed from Pubmed, Medline, and Cochrane databases for articles published from their inception to October 30, 2020. We performed a chi-squared analysis of homogenized data to examine the relationship between recurrence location and rate of recurrence. A risk of bias was obtained with the ROBINS-I tool. [PROSPERO ID: CRD42020221826]

Results: Among the included studies, there were a total of $15(0.52 \%)$ local recurrences of melanoma in-situ and invasive melanoma after MMS with MART-1.

Conclusions: This review has served to demonstrate that MMS with MART-1 immunostaining in frozen sections is a technique that produces satisfactory recurrence rates for melanoma in-situ and invasive melanoma.

\section{INTRODUCTION}

Cutaneous melanoma (CM) affects about 287,000 people each year, worldwide. ${ }^{1}$ Worse prognoses are associated with $\mathrm{CM}$ located on the head, neck, and trunk compared to extremities - with the exception of acral melanoma. ${ }^{2}$ Surgical management continues to be the primary therapeutic approach for $\mathrm{CM}^{3}$ Current surgical recommendations for primary $\mathrm{CM}$ include surgical excision with pre-determined surgical margins based on tumor characteristics and subsequent microscopic margin assessment - as needed. Wide local excision (WLE) continues to be the primary recommendation of the American Academy of Dermatology (AAD), with margins predicated by tumor depth. ${ }^{3}$ Extra consideration must be made for anatomically constrained sites and/or when subclinical spread is suspected. Mohs micrographic surgery (MMS) is recommended to treat primary melanoma in situ and lentigo maligna located on the head and neck, acral sites, genitalia, and pretibial leg, and for locally recurrent melanoma in situ lentigo maligna in any location. ${ }^{4}$ The 2019 AAD workgroup has encouraged further study of MMS on these anatomically constrained areas.

Mohs micrographic surgery offers particular advantages for tumors located in areas that require tissue conservation and when subclinical spread is suspected. MMS is ideal for the head and neck due to the high 
recurrence rates of $\mathrm{CM}$ due to particular risk features, such as, subclinical spread, larger preoperative size, more likely to have had previous treatment, and requiring tissuerearranging reconstruction when compared to keratinocytic tumors. ${ }^{5}$ Immediate margin assessment provides the confidence of complete tumor removal. Unfortunately, hematoxylin and eosin frozen sections are notoriously difficult to interpret for $\mathrm{CM}$, resulting in poor sensitivity and specificity. ${ }^{6-}$ 12 Over the years, immediate margin assessment has evolved to a more specific analysis. The utilization of immunostains, such as melanoma-associated antigen recognized by T-cells (MART- 1 ), has improved the confidence in and histologic accuracy of MMS in some subtypes of CM. ${ }^{13-15}$ This is most evident in melanocytic lesions demonstrating extensive subclinical spread. ${ }^{14-21}$ Furthermore, MART-1 staining of frozen sections has become more rapid and as reliable as permanent sections. ${ }^{13,22} \mathrm{~A}$ recent survey of Mohs surgeons and database analysis suggests wide-spread use of MART-1 in MMS for CM. ${ }^{23,24}$

With techniques in MMS for treating melanocytic lesions evolving, there is an impetus to continue to evaluate emerging research for further clinical guideline development. To date, no systematic reviews have specifically examined the utilization of MART-1 staining in frozen sections for CM - particularly its effect on local recurrence. Sharma and colleagues ${ }^{25}$ performed a broad systematic review to compare the local recurrence of MMS with that of traditional surgical techniques for lentigo maligna/lentigo maligna melanoma (LM/LMM) but only included 2 studies and failed to compare with MART-1.

In this study, the authors aim to systematically review and analyze the rate of local recurrence after utilization of Mohs micrographic surgery with MART-1 immunohistochemistry in frozen sections in patients being treated for primary and recurrent cutaneous melanoma. The increasing utilization of MART-1 staining in MMS supports the further evaluation of the practice.

\section{METHODS}

The authors followed the guidelines of the Cochrane Handbook for Systematic Reviews of Interventions to execute the study. The authors followed the PRISMA (Preferred Reporting Items for Systematic Reviews and Meta-analyses) statement and checklist to publish and report this metaanalysis. The protocol was determined before the search and data extraction took place. [PROSPERO ID: CRD42020221826]

\section{Search Strategy}

The authors conducted a systematic search with Pubmed, Medline, and Cochrane databases for articles published from their inception to October 30,2020, to identify relevant studies published in English. Electronic searches were performed with the following search terms: ["Mohs" AND "Melanoma"]. The ClinicalTrials.gov database was searched for current trials. The authors manually checked the bibliographies of the identified articles, including any relevant reviews or metaanalyses to further identify eligible studies.

\section{Eligibility Criteria}

The inclusion criteria are (1) Utilization of MART-1 immunohistochemistry in frozen sections, (2) melanoma in situ or invasive melanoma, (3) prospective, retrospective, (5) randomized controlled trials (6) published in a peer-reviewed 
journal, (7) published in English, (8) and fulltext of the studies available. The exclusion criteria are (1) digital melanoma, (2) "how we do it" articles, (3) review studies (4), and basic science studies.

\section{Data Extraction and Outcomes}

All relevant information regarding study characteristics - design, level of evidence, the methodological quality of evidence, population, outcome measures, and followup time points - were collected by two independent reviewers using a predetermined datasheet. Two independent reviewers oversaw all review process steps, and data were independently obtained and their inclusion criteria agreed upon. Any disagreement over data relevancy were settled by a third reviewer. The primary extracted outcome was local recurrence rates.

\section{Quality Assessment}

Patient characteristics and control protocols were reviewed to evaluate for possible confounding variables. The Risk-of-bias (RoB) plot was created using The Cochrane Risk of Bias in Non-randomized Studies of Interventions (ROBINS-I) tool. RoB judgments were made following the supplementary guidance of Sterne et. al. ${ }^{26}$

\section{Data Analysis}

The number of local recurrences was homogenized by determining the percent affected. A $X^{2}$ test of independence was performed to examine the relationship between recurrence location and rate of recurrence. A comparison of proportions was utilized to analyze demographic data. A P-value $<0.05$ was considered statistically significant, and $95 \%$ confidence intervals (Cls) were reported.

\section{RESULTS}

\section{Literature Search}

The literature search identified 617 total manuscripts. Once articles were screened for inclusion and exclusion criteria, 17 fulltext articles were assessed for eligibility (Figure 1).

Four manuscripts with a total of 2,896 lesions were included in the review. ${ }^{17,18,27,28}$ Nine manuscripts were excluded due to the lack of recurrence rates. ${ }^{4,13-16,20,29-31}$ Single manuscripts were excluded due to the lesion type ${ }^{32}$, modified procedures $^{19}$, digital melanoma ${ }^{33}$, or inclusion of the same data set. ${ }^{21}$ Clinicaltrials.gov did not provide any additional information concerning on-going research on this subject.

\section{Study Characteristics}

All four included studies, summarized in Table 1, were retrospective. There were 492 invasive melanomas (IM) and 2,404 melanoma in-situ (MIS), of which 2,556 were primary lesions. Of the included lesions, 1,724 were located on the head/neck and 1,172 were located on the trunk/extremities. The outcomes utilized in the included studies were local recurrence, rate of metastasis, overall survival, disease-free survival rates, upstaging of the lesion, and/or surgical margins required for complete removal. Mean follow-up periods ranged from 1,026 to 1,361 days, with a reported minimum and maximum of 3 and 3167 days, respectively.

\section{Demographics}

Age was reported as a mean with a range in all of the included studies. There was no report of standard deviation. The ages of the 
patients in this review ranged from 12 to 99. The average age of participants was 66 in three of the four included studies, and not reported in the last.

Sex demographics were reported in all of the included studies. A (chi-squared test) significant majority of the participants were male, with a total of $61.5 \%$ [P $<0.0001]$.

The location of the lesions was reported in all of the included studies. While some studies were more specific with particular locations, all of them included head, neck, trunk, and limbs. In total, 1,724 melanomas were on the head and neck; 1,172 were elsewhere (Table 1).

Tumor specifics included whether or not the lesion was primary or recurrent and invasive or in-situ. The average Breslow depth of invasive melanomas was also included. There were 2,556 (88\%) primary melanomas. Of these melanomas, 2,404 $(83 \%)$ were MIS. The average Breslow depth (of IM) was estimated to be $0.768 \mathrm{~mm}$ with a range of $0.1-5.8 \mathrm{~mm}$. Two studies did not report the range of depth, and one did not provide an average depth.

\section{Methodology}

All of the included studies were retrospective cohort studies that gathered data from their respective institutions only. The MMS technique described varied slightly. Two of the studies report utilizing a central debulking excision for bread-loafing in permanent paraffin-embedded H\&E for the staging of the melanoma. ${ }^{17,28}$ The remaining studies either did not report such a technique or were vague about their technique, merely referring to previous reports. Two studies described an initial debulking margin of $2-3 \mathrm{~mm}$ with an additional $2-3 \mathrm{~mm}$ Mohs margin that was subsequently utilized for frozen sections. ${ }^{17,28}$ Degesys et al. reported an initial $5 \mathrm{~mm}$ margin with $3 \mathrm{~mm}$ subsequent Mohs margins. ${ }^{27}$ Valentin et al. did not report the specific margins utilized. ${ }^{18}$ Positive margin determination was relatively homogenous across the studies. They all utilized, at a minimum, the following: pagetoid spread, nests of at least 3 atypical melanocytes, and confluent melanocyte hyperplasia along the basement membrane. Follow-up data was collected similarly across the included studies. With the exception of one, all of the included studies utilized over-the-phone screening of the participants.

\section{Local Recurrence}

Among the included studies, there were 15 $(0.52 \%)$ local recurrences of melanoma in situ and invasive melanoma after MMS with MART-1. The descriptions of each recurrence can be found in Table 2. MIS did not convert to IM in any of the reported recurrences. In primary vs recurrent melanomas (IM and MIS), $X^{2}(1, N=2,894)$ $=3.26, p=.071$, the chance of recurrence was not significantly different. By contrast, the chance of recurrence was significantly higher in invasive melanoma when compared to melanoma in situ: $X^{2}(1, N=$ $2,896)=19.76, p<.001$. There was no significant difference in $\mathrm{CM}$ recurrence between the two general locations of "head/neck" and "other location": $X^{2}(1, N=$ $2,896)=2.65, p=0.10$. Notably, all "other location" recurrences were on the feet; no recurrences were observed on the proximal extremities. Of the head and neck melanomas, most local recurrence (1.99\%) occurred on the scalp. All were initially invasive melanomas.

\section{Risk of Bias}


The review authors judged that three studies had a moderate RoB and the final study had a low RoB (Figure 2). All of the included studies were able to measure important known confounding domains, such as, patient age, patient sex, tumor location, Breslow depth, primary and recurrent lesions, and lesion type. The cohort research design without the use of control groups suggests that the effect of confounding variables within the treatment groups is unlikely. Bias in the selection of participants into the study was moderate for Degesys et. al. ${ }^{27}$ due to risk of recall bias in 6 -month telephone follow-up. Foxton et. al. ${ }^{17}$

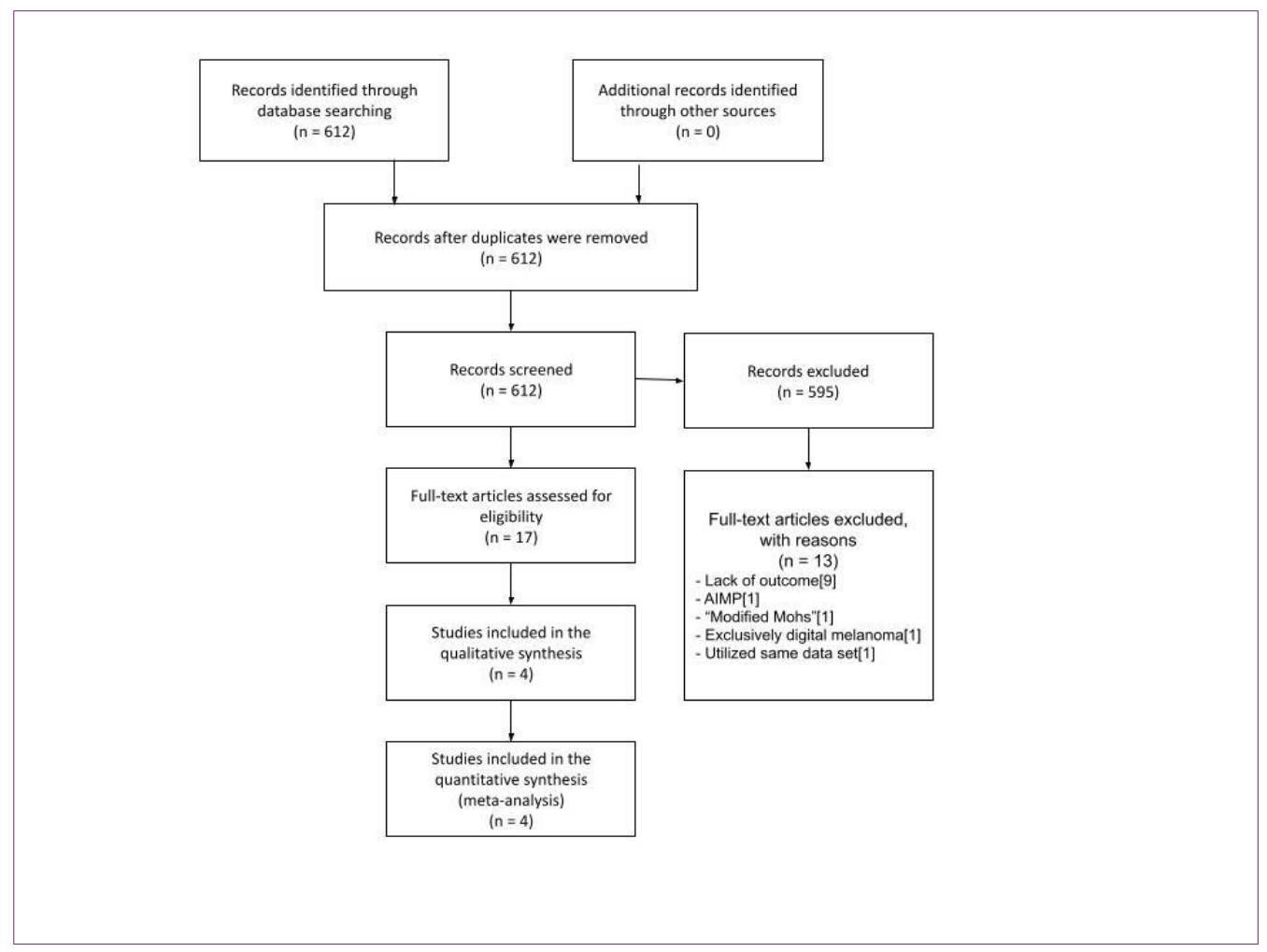

Figure 1. PRISMA Flow-Chart

AIMP; atypical intraepidermal melanocytic proliferation 


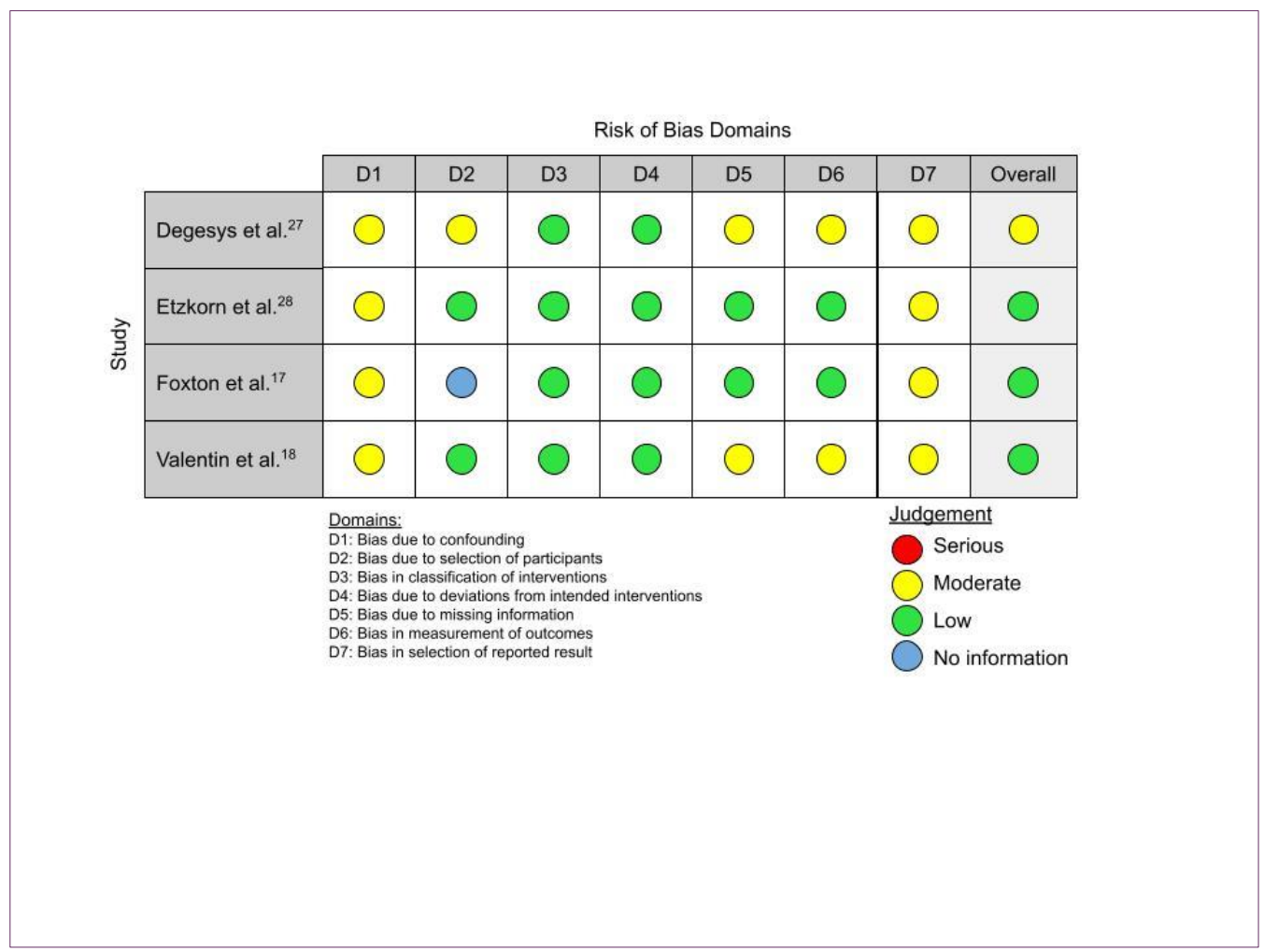

Figure 2. Risk of Bias Analysis

provided no information about the selection of participants or the database they used. Degesys et. al. lost $13.9 \%$ of patients to follow up and Valentin et. al. lost $24.2 \%$ of patients to follow up and were considered to have a moderate risk of bias due to missing data.

\section{DISCUSSION}

MMS with MART-1 demonstrates lower recurrence rates than conventional excision or MMS without MART-1. ${ }^{34}$ Of the included studies, the rate of recurrence of MIS after MMS with MART-1 was just 0.25\% (6/2404). According to the work of Nosrati et. al., if the MART-1 stain is not used with MMS, the recurrence rate increases to $1.8 \%(5 / 277)$.

The recurrence rate of MIS after WLE has been reported to be as high as 5.7\%
(22/395). ${ }^{35}$ The utilization of MART-1 in MMS for CM demonstrates advantages over classical MMS in recurrence ${ }^{27,36}$ and in surgeon preference.

The transition to MART-1 from H\&E alone and/or HMB-45 took place in many important retrospective studies. ${ }^{14,20,21,33,36,37}$ This transition appeared to occur around 2002, when further investigation suggested MART-1 was the superior immunohistochemistry stain in frozen sections due to reliable epidermal staining, ease of interpretation, and highest histopathologic concordance. ${ }^{14,15,38-41}$ Subsequently, studies began to demonstrate improved recurrence rates after transitioning to exclusively using MART-1.21,33,36 In a recent study by Burnett et al., ${ }^{37}$ a retrospective analysis of IM on the trunk and proximal extremities treated by MMS observed only $2(0.14 \%)$ recurrences. The authors established that the sample included procedures that utilized $\mathrm{H} \& \mathrm{E}$ alone, then 
HMB, then MART-1 exclusively. Recurrences occurred only prior to the incorporation of MART-1 immunostaining in MMS. While the sample included in this study was much larger than the invasive melanoma collective sample in the present study, it corroborates an extremely low recurrence rate when MART- 1 is utilized for IM for the trunk and proximal extremities.

From 2001 to 2016, the chances of receiving MMS for melanoma increased 304\%. Twenty-seven percent of the MMS cases reported use of Immunohistochemistry stains (IHC), with an increase in utilization over recent years with further acceptance of its benefit. ${ }^{24}$ In addition to ease of use/interpretability and outcome improvement, it is important to investigate financial feasibility. Wilson et al. demonstrated that among comprehensive margin assessment techniques for MIS/IM, staged excision using en-face margin assessment (SEEM) and MMS with MART-1 were similar in cost for the healthcare system. The authors noted that SEEM incurred lower costs due to decreased excision code reimbursement and lower number of stages, while MMS with MART-1 required less complex reconstruction and the multiple procedure reduction rule. MMS "classic" with H\&E generally required more stages, larger margins, and a flap or graft. ${ }^{31}$

Recent epidemiological research suggests that there are no significant differences in local recurrence or overall survival when comparing MMS and WLE for head, neck, trunk, and extremities. ${ }^{42-44}$ Conversely, Valentin et al. provides 5-year Kaplan-Meier local recurrence rates for primary $\mathrm{CM}$ treated with MMS with MART-1 that are significantly lower than historical controls of conventional wide-local excision. ${ }^{18}$ We feel that these discordances may have occurred due to lack of considering specific MM

Table 1: Study Characteristics

\begin{tabular}{|c|c|c|c|c|c|c|c|c|}
\hline & Foxton & Degesys & Etzkorn & Valentin & Total & & & \\
\hline $\mathbf{n}$ & 62 & 123 & 597 & 2,114 & 2896 & & & \\
\hline $\begin{array}{l}\text { Age, } \\
\text { (range) }\end{array}$ & $63.2(34-88)$ & $\begin{array}{l}66.48 \quad(17- \\
94)\end{array}$ & $66(18-93)$ & $66(12-99)$ & & & & \\
\hline \multicolumn{9}{|l|}{ Sex } \\
\hline Female & 33 (53.2\%) & 45 (36.6\%) & 226 (37.9\%) & $810(38.3 \%)$ & 1114 & $38.50 \%$ & & $P<0.0001$ \\
\hline Male & $29(46.8 \%)$ & $78(63.4 \%)$ & $371(62.1 \%)$ & $\begin{array}{l}1304 \\
(61.7 \%)\end{array}$ & 1782 & $61.50 \%$ & & \\
\hline Lesion type & & & & & & LR & LR\% & \\
\hline Primary & 58 (93.5) & $117(95.1)$ & 499 (83.6) & $1882(89.1)$ & 2556 & 11 & $0.43 \%$ & $P=0.0710$ \\
\hline \multirow[t]{2}{*}{ Recurrent } & $4(6.5)$ & $6(4.9)$ & $98(16.4)$ & 230 (10.9) & 338 & 4 & $1.18 \%$ & \\
\hline & & & & & & LR & LR\% & \\
\hline IM & 8 (12.9) & $123(100)$ & $161(27)$ & $200(9.5)$ & 492 & 9 & $1.83 \%$ & $P<0.0001$ \\
\hline Desmoplastic & NR & NR & 8 & NR & & & & \\
\hline MIS & $54(87.1)$ & 0 & $436(73)$ & $1,914(90.5)$ & 2404 & 6 & $0.25 \%$ & \\
\hline Location & & & & & & LR & LR\% & \\
\hline Other location & $7(11.3)$ & $53(43.1)$ & 122 & 990 & 1172 & 3 & $0.25 \%$ & $P=0.1035$ \\
\hline $\begin{array}{l}\text { Head/Neck, } \\
\text { total }\end{array}$ & $55(88.7)$ & $70(56.9)$ & 475 (79.4) & 1124 (53.2) & 1724 & 12 & $0.69 \%$ & \\
\hline Face & 49 & 47 (38.2) & 355 (74.7) & $944(44.7)$ & 1395 & 9 & $0.64 \%$ & \\
\hline Neck & 3 & $11(8.9)$ & $26(5.5)$ & $82(3.9)$ & 122 & 0 & 0 & \\
\hline Scalp & 3 & $5(4.1)$ & $45(9.5)$ & $98(4.6)$ & 151 & 3 & $1.99 \%$ & \\
\hline Ears & NR & $7(5.7)$ & 49 (10.3) & NR & 56 & 0 & 0 & \\
\hline \multicolumn{9}{|l|}{$\begin{array}{l}\text { Local } \\
\text { recurrence data }\end{array}$} \\
\hline Mean F/U time & (3-30 mo) & 1273 days & 1026 days & 3.73 years & & & & \\
\hline LR, total & 0 & $2(1.63 \%)$ & $2(0.34 \%)$ & $11(0.52 \%)$ & & & & \\
\hline
\end{tabular}

November 2021 Volume 5 Issue 6 
Table 2: Description of Recurrences

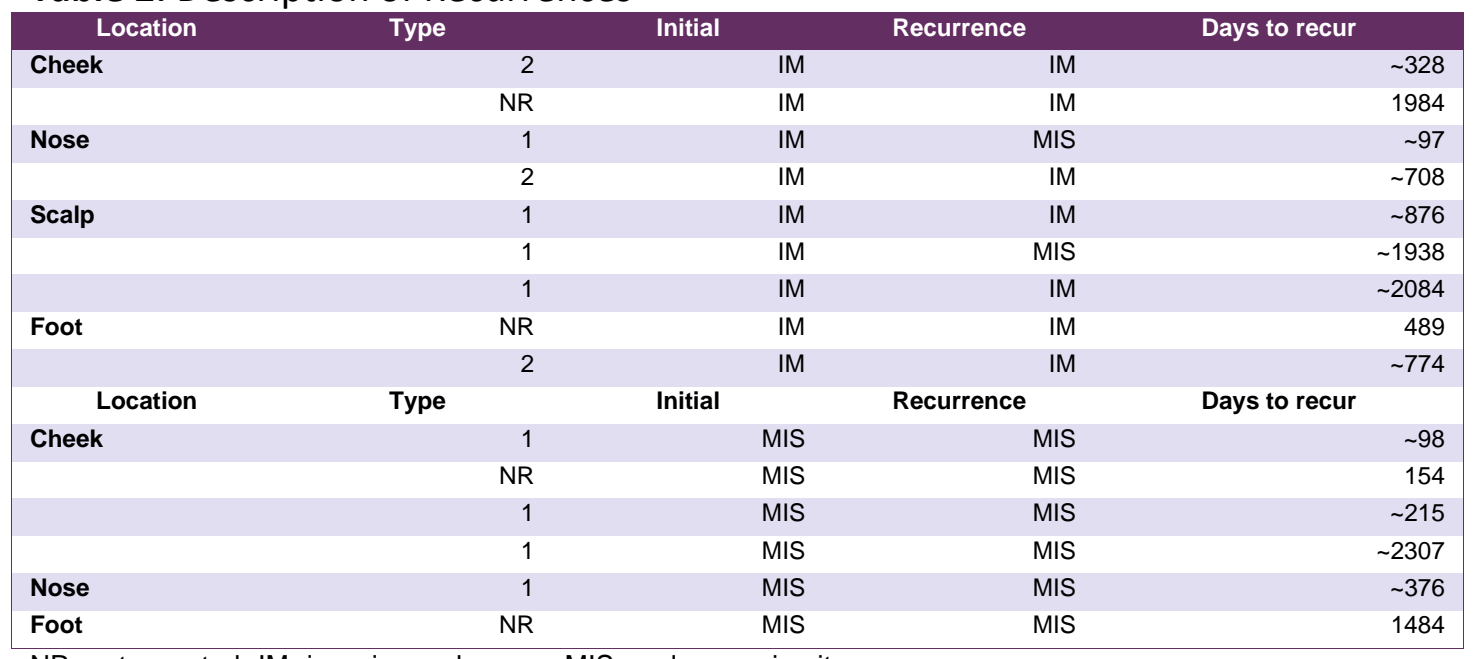

$\mathrm{NR}$; not reported. IM; invasive melanoma. MIS; melanoma in-situ. 1; Primary. 2; Secondary

techniques as a confounding variable in epidemiological studies.

The data presented in this study demonstrates that the increasing utilization of MART-1 in MMS for cutaneous melanoma is warranted and should be recommended, where applicable. The evidence supports current $\mathrm{AUC}^{3}$ and $\mathrm{NCCN}^{45}$ recommendations while evidence suggests further use of MMS with MART-1 for CM where local excision continues to demonstrate worse recurrence rates. MMS with MART-1 has demonstrated strong evidence to support its routine use for invasive melanoma at "special sites" (head, neck, acral sites, genitalia, and pretibial leg) and on the trunk/proximal extremities.

The limitations of this review and the included studies are inherent and technical. The primary limitation of the included studies is that they were all observational studies that were retrospective in nature. A limitation of the review was the exclusion of alternative surgical techniques utilizing

MART-1, such as modified staged-excision. Furthermore, there may be a serious confounding variability in surgical technique, frozen section technique, and MART-1 interpretation skill. Lastly, this review did not differentiate the lentigo maligna and melanoma in situ as separate entities and were all treated as MIS.

\section{CONCLUSION}

This review demonstrates that the technique of MMS with MART-1 IHC in frozen sections produces satisfactory recurrence rates for melanoma in situ and invasive melanoma. There appears to be discordance between current guidelines and research supporting broader use of MMS with MART-1. Further prospective research should be done to evaluate clinical outcomes with the use of MMS for invasive and noninvasive CM in various body locations.

Conflict of Interest Disclosures: None

Funding: None

Corresponding Author:

Tyler Long, DO

Dermatology Resident

River Ridge Dermatology

HCA Healthcare LewisGale Hospital-Montgomery

Phone: 540-520-6082

November $2021 \quad$ Volume 5 Issue 6 
Email: Tyler.Long@hcahealthcare.com

\section{References:}

1. Ferlay, J. et al. Estimating the global cancer incidence and mortality in 2018: GLOBOCAN sources and methods. Int. J. Cancer 144, 19411953 (2019).

2. Balch, C. M. Cutaneous melanoma: prognosis and treatment results worldwide. Semin. Surg. Oncol. 8, 400-414 (1992).

3. Swetter, S. M. et al. Guidelines of care for the management of primary cutaneous melanoma. J. Am. Acad. Dermatol. 80, 208-250 (2019).

4. Etzkorn, J. R. et al. Correlation Between Appropriate Use Criteria and the Frequency of Subclinical Spread or Reconstruction With a Flap or Graft for Melanomas Treated With Mohs Surgery With Melanoma Antigen Recognized by T Cells 1 Immunostaining. Dermatol. Surg. 42, 471-476 (2016).

5. Fix, W. et al. Melanomas of the head and neck have high-local recurrence risk features and require tissue-rearranging reconstruction more commonly than basal cell carcinoma and squamous cell carcinoma: A comparison of indications for microscopic margin control prior to reconstruction in 13,664 tumors. J. Am. Acad. Dermatol. 85, 409-418 (2021).

6. Stonecipher, M. R., Leshin, B., Patrick, J. \& White, W. L. Management of lentigo maligna and lentigo maligna melanoma with paraffinembedded tangential sections: utility of immunoperoxidase staining and supplemental vertical sections. J. Am. Acad. Dermatol. 29, 589-594 (1993).

7. Dhawan, S. S., Wolf, D. J., Rabinovitz, H. S. \& Poulos, E. Lentigo maligna. The use of rush permanent sections in therapy. Arch. Dermatol. 126, 928-930 (1990).

8. Prieto, V. G. et al. Are en face frozen sections accurate for diagnosing margin status in melanocytic lesions? Am. J. Clin. Pathol. 120, 203-208 (2003).

9. Barlow, R. J., White, C. R. \& Swanson, N. A. Mohs' micrographic surgery using frozen sections alone may be unsuitable for detecting single atypical melanocytes at the margins of melanoma in situ. Br. J. Dermatol. 146, 290294 (2002).

10. Cohen, L. M., McCall, M. W. \& Zax, R. H. Mohs micrographic surgery for lentigo maligna and lentigo maligna melanoma. A follow-up study. Dermatol. Surg. 24, 673-677 (1998).
11. Cohen, L. M. et al. Successful treatment of lentigo maligna and lentigo maligna melanoma with Mohs' micrographic surgery aided by rush permanent sections. Cancer 73, 2964-2970 (1994).

12. Bene, N. I., Healy, C. \& Coldiron, B. M. Mohs micrographic surgery is accurate $95.1 \%$ of the time for melanoma in situ: a prospective study of 167 cases. Dermatol. Surg. 34, 660-664 (2008).

13. Kelley, L. C. \& Starkus, L. Immunohistochemical staining of lentigo maligna during Mohs micrographic surgery using MART-1. J. Am. Acad. Dermatol. 46, 78-84 (2002).

14. Zalla, M. J., Lim, K. K., Dicaudo, D. J. \& Gagnot, M. M. Mohs micrographic excision of melanoma using immunostains. Dermatol. Surg. 26, 771-784 (2000).

15. Albertini, J. G., Elston, D. M., Libow, L. F., Smith, S. B. \& Farley, M. F. Mohs micrographic surgery for melanoma: a case series, a comparative study of immunostains, an informative case report, and a unique mapping technique. Dermatol. Surg. 28, 656-665 (2002).

16. Ellison, P. M., Zitelli, J. A. \& Brodland, D. G. Mohs micrographic surgery for melanoma: $A$ prospective multicenter study. J. Am. Acad. Dermatol. 81, 767-774 (2019).

17. Foxton, G. C., Elliott, T. G. \& Litterick, K. A. Treating melanoma in situ and lentigo maligna with Mohs micrographic surgery in Australia. Australas. J. Dermatol. 60, 33-37 (2019).

18. Valentín-Nogueras, S. M., Brodland, D. G., Zitelli, J. A., González-Sepúlveda, L. \& Nazario, C. M. Mohs Micrographic Surgery Using MART1 Immunostain in the Treatment of Invasive Melanoma and Melanoma In Situ. Dermatol. Surg. 42, 733-744 (2016).

19. Heath, M., Woody, M., Leitenberger, J., Latour, E. \& Bar, A. Invasive Melanoma and Melanoma in Situ Treated With Modified Mohs Micrographic Surgery With En Face Permanent Sectioning: A 10-Year Retrospective Review. Dermatol. Surg. 46, 1004-1013 (2020).

20. Kunishige, J. H., Doan, L., Brodland, D. G. \& Zitelli, J. A. Comparison of surgical margins for lentigo maligna versus melanoma in situ. J. Am. Acad. Dermatol. 81, 204-212 (2019).

21. Stigall, L. E., Brodland, D. G. \& Zitelli, J. A. The use of Mohs micrographic surgery (MMS) for melanoma in situ (MIS) of the trunk and proximal extremities. J. Am. Acad. Dermatol. 75, 1015-1021 (2016).

22. Cherpelis, B. S., Moore, R., Ladd, S., Chen, R. \& Glass, L. F. Comparison of MART-1 frozen sections to permanent sections using a rapid November $2021 \quad$ Volume 5 Issue 6 
19-minute protocol. Dermatol. Surg. 35, 207213 (2009).

23. Siscos, S. M., Neill, B. C., Seger, E. W., Hooton, T. A. \& Hocker, T. L. H. The Current State of Mohs Surgery for the Treatment of Melanoma: A Nationwide Cross-Sectional Survey of Mohs Surgeons. Dermatol. Surg. 46, 1267-1271 (2020).

24. Lee, M. P. et al. Evolution of Excisional Surgery Practices for Melanoma in the United States. JAMA Dermatol. 155, 1244-1251 (2019).

25. Sharma, A. N., Foulad, D. P., Doan, L., Lee, P. K. \& Atanaskova Mesinkovska, N. Mohs surgery for the treatment of lentigo maligna and lentigo maligna melanoma - a systematic review. $J$. Dermatolog. Treat. 32, 157-163 (2021).

26. Sterne, J. A. et al. ROBINS-I: a tool for assessing risk of bias in non-randomised studies of interventions. BMJ 355, i4919 (2016).

27. Degesys, C. A., Powell, H. B., Hsia, L.-L. B. \& Merritt, B. G. Outcomes for Invasive Melanomas Treated With Mohs Micrographic Surgery: A Retrospective Cohort Study. Dermatol. Surg. 45, 223-228 (2019).

28. Etzkorn, J. R. et al. Low recurrence rates for in situ and invasive melanomas using Mohs micrographic surgery with melanoma antigen recognized by T cells 1 (MART-1) immunostaining: tissue processing methodology to optimize pathologic staging and margin assessment. J. Am. Acad. Dermatol. 72, 840850 (2015).

29. Shin, T. M. et al. Clinical and pathologic factors associated with subclinical spread of invasive melanoma. J. Am. Acad. Dermatol. 76, 714-721 (2017).

30. Shin, T. M. et al. Clinical factors associated with subclinical spread of in situ melanoma. J. Am. Acad. Dermatol. 76, 707-713 (2017).

31. Wilson, J. G., German, R., Hamann, C. R., Vidal, N. Y. \& LeBoeuf, M. Comparison of staged excision and Mohs micrographic surgery with and without MART-1 immunostains for surgical treatment of melanoma of the head, neck, and special sites: A retrospective cohort study. J. Am. Acad. Dermatol. 84, 192-194 (2021).

32. Etzkorn, J. R. et al. Mohs micrographic surgery with melanoma antigen recognized by T cells 1 (MART-1) immunostaining for atypical intraepidermal melanocytic proliferation. J. Am. Acad. Dermatol. 79, 1109-1116.e1 (2018).

33. Terushkin, V., Brodland, D. G., Sharon, D. J. \& Zitelli, J. A. Digit-Sparing Mohs Surgery for Melanoma. Dermatol. Surg. 42, 83-93 (2016).

34. Rzepecki, A. K. et al. The 'Rule of 10s' versus the 'Rule of 2s': high complication rates after conventional excision with postoperative margin assessment of specialty site versus trunk and proximal extremity melanomas. J. Am. Acad. Dermatol. (2018).

35. Nosrati, A. et al. Outcomes of Melanoma In Situ Treated With Mohs Micrographic Surgery Compared With Wide Local Excision. JAMA Dermatol. 153, 436-441 (2017).

36. Bricca, G. M., Brodland, D. G., Ren, D. \& Zitelli, J. A. Cutaneous head and neck melanoma treated with Mohs micrographic surgery. J. Am. Acad. Dermatol. 52, 92-100 (2005).

37. Burnett, M. E., Brodland, D. G. \& Zitelli, J. A. Long-term outcomes of Mohs micrographic surgery for invasive melanoma of the trunk and proximal portion of the extremities. J. Am. Acad. Dermatol. 84, 661-668 (2021).

38. Semkova, K., Mallipeddi, R., Robson, A. \& Palamaras, I. Mohs micrographic surgery concordance between Mohs surgeons and dermatopathologists. Dermatol. Surg. 39, 16481652 (2013).

39. Highsmith, J. T., Highsmith, M. J. \& Monheit, G. D. Histologic Accuracy of Mohs Micrographic Surgery. Dermatol. Surg. 44, 350-353 (2018).

40. Kesty, K., Sangueza, O. P., Leshin, B. \& Albertini, J. G. Mohs micrographic surgery and dermatopathology concordance; An analysis of 1421 Mohs cases over 17 years. J. Am. Acad. Dermatol. (2017) doi:10.1016/j.jaad.2017.11.055.

41. Mariwalla, K., Aasi, S. Z., Glusac, E. J. \& Leffell, D. J. Mohs micrographic surgery histopathology concordance. J. Am. Acad. Dermatol. 60, 94-98 (2009).

42. Demer, A. M., Hanson, J. L., Maher, I. A. \& Liszewski, W. Association of Mohs Micrographic Surgery vs Wide Local Excision With Overall Survival Outcomes for Patients With Melanoma of the Trunk and Extremities. JAMA Dermatol. 157, 84-89 (2021).

43. Phan, K., Onggo, J. \& Loya, A. Mohs micrographic surgery versus wide local excision for head and neck melanoma-in-situ. J. Dermatolog. Treat. 31, 559-562 (2020).

44. Daniel, V. T., Crawford, A., Kiefe, C. I. \& Mahmoud, B. H. Recurrence and Mortality of Melanoma In Situ of the Trunk or Extremities: A Surveillance, Epidemiology, and End Results Analysis. Dermatol. Surg. 47, 1-5 (2021).

45. National Comprehensive Cancer Network. SpringerReference (2012) doi:10.1007/springerreference_178671.

November $2021 \quad$ Volume 5 Issue 6 
